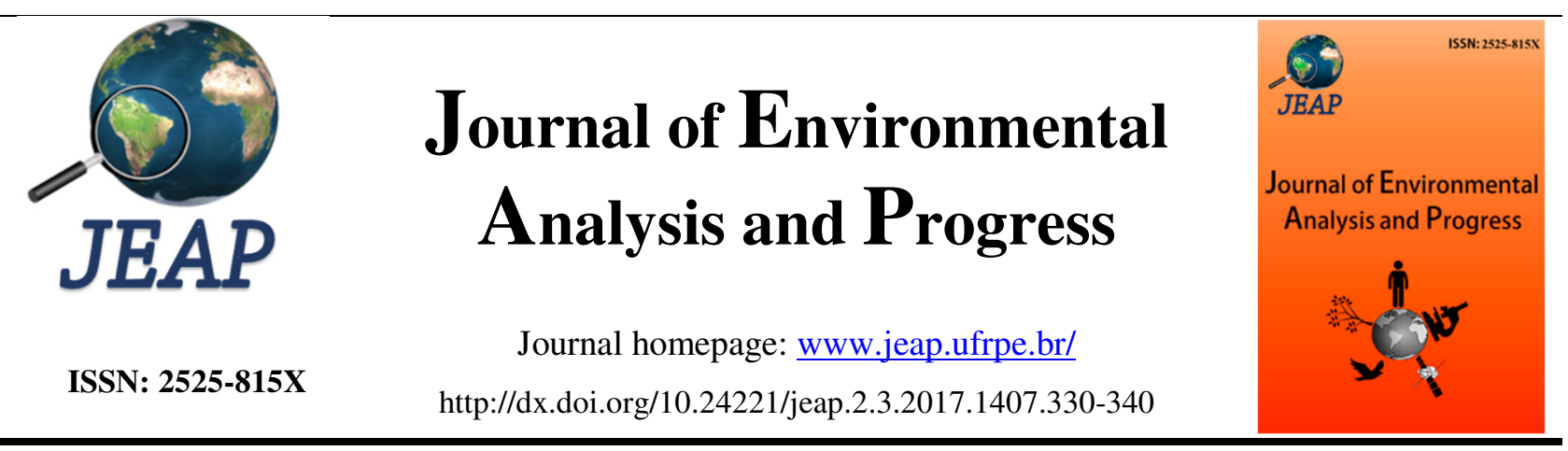

\title{
Potencial de geração de energia eólica no submédio São Francisco
}

\section{Wind power generation potential in the San Francisco Submiddle}

\author{
Iug Lopes ${ }^{\mathrm{a}}$, Euvaldo Pereira de Cerqueira Júnior ${ }^{\mathrm{b}}$, Juliana Maria Medrado de Melo ${ }^{\mathrm{a}}$, Brauliro \\ Gonçalves Leal ${ }^{\mathrm{b}}$
}

${ }^{\text {a }}$ Universidade Federal Rural de Pernambuco, Doutorando do Programa de Pós-Graduação em Engenharia Agrícola. Rua Dom Manoel de Medeiros, s/n, Dois Irmãos, Recife-PE. CEP: 52.171-900. E-mail: iuglopes@hotmail.com; medrado.juliana@gmail.com.

b Universidade Federal do Vale do São Francisco-UNIVASF. Mestrando do Programa de Pós-Graduação em Engenharia Agrícola. Avenida Antonio Carlos Magalhães, n. 510, Country Club, Juazeiro-BA. CEP: 48.9002-300. E-mail: euvaldojr.engagronomo@gmail.com; brauliro.leal@gmail.com.

\begin{tabular}{|c|c|}
\hline A R T I C L E I N F O & A B S T RA C T \\
\hline $\begin{array}{l}\text { Recebido 21 Jun } 2017 \\
\text { Aceito 29 Jul } 2017 \\
\text { Publicado } 31 \text { Jul } 2017\end{array}$ & $\begin{array}{l}\text { The major potential of wind power generation in the world and a possibility of } \\
\text { electric energy production make it an alternative source to increase the diversity in } \\
\text { the world energy matrix. The objective was to characterize a predominant velocity } \\
\text { and direction of the wind for generation of wind power in the Submédio São } \\
\text { Francisco. Wind speed }\left(\mathrm{m}^{-1} \mathrm{~s}^{-1}\right) \text {, direction }\left({ }^{\circ}\right) \text { and the wind }\left(\mathrm{m}^{-1} \mathrm{~s}^{-1}\right) \text { wind speed } 10 \mathrm{~m} \\
\text { high, air temperature }\left({ }^{\circ} \mathrm{C}\right) \text {, and atmospheric pressure }(\mathrm{hPa}) \text { were used. Map of } \\
\text { velocity }\left(\mathrm{m} \cdot \mathrm{s}^{-1}\right) \text {, direction }\left({ }^{\circ}\right) \text {, the wind }\left(\mathrm{m}^{-1} \mathrm{~s}^{-1}\right) \text { of wind; And also of the wind potential } \\
\left(\mathrm{W} \cdot \mathrm{m}^{-2}\right) \text { of the São Francisco Submiddle. The highest values of wind power are } \\
\text { highlighted for the municipalities of Dormentes, Lagoa Grande, Curaçá, Petrolina, } \\
\text { Afrânio, Pedro Alexandre, part of the municipality of Santa Filomena, Santa Maria } \\
\text { da Boa Vista, Chorrochó, Macurure, Paulo Afonso, and Juazeiro. The municipality } \\
\text { of Curaçá, in the State of Bahia, show the most favorable installation of wind } \\
\text { generators, instead of larger values and constant wind transmission. Consequently, it } \\
\text { has the highest values of wind energy and a dominant frequency in the southeast } \\
\text { direction of the winds throughout the day, being able to fix the towers in this } \\
\text { geographical orientation. A large part of the Submédio São Francisco is suitable for } \\
\text { the installation of wind energy generation centers. }\end{array}$ \\
\hline
\end{tabular}

Keywords: Meteorological stations, wind turbines, semi-arid.

\section{R E S U M O}

O grande potencial de geração de energia eólica no mundo e a possibilidade de geração de energia elétrica fazem dela uma fonte alternativa para aumentar a diversidade na matriz energética mundial. O objetivo foi caracterizar a velocidade ea direção predominante do vento para geração de energia eólica no Submédio São Francisco. Utilizaram-se dados de velocidade por hora $\left(\mathrm{m} \cdot \mathrm{s}^{-1}\right)$, direção $\left(^{\circ}\right)$ e vento $\left(\mathrm{m}_{\mathrm{s}} \mathrm{s}^{-1}\right)$ vento $10 \mathrm{~m}$ de altura, temperatura do ar $\left({ }^{\circ} \mathrm{C}\right)$ e pressão atmosférica $(\mathrm{hPa})$ medida em 37 estações meteorológicas automáticas. Foram apresentados mapas de velocidade $\left(\mathrm{m} . \mathrm{s}^{-1}\right)$, direção $\left(^{\circ}\right)$, vento $\left(\mathrm{m} . \mathrm{s}^{-1}\right)$ do vento e, também, do potencial eólico $\left(\mathrm{W} . \mathrm{m}^{-2}\right)$ do Submédio São Francisco. Os maiores valores de potência eólica estão destacados para os municípios de Dormentes, Lagoa Grande, Curaçá, Petrolina, Afrânio, Pedro Alexandre, parte do município de Santa Filomena, Santa Maria da Boa Vista, Chorrochó, Macururé, Paulo Afonso e Juazeiro. O município de Curaçá, no estado da Bahia, apresenta a instalação mais favorável de geradores eólicos, devido aos maiores valores e velocidade constante dos ventos. Consequentemente, 
apresenta um dos maiores produtores de energia eólica, com uma frequência predominante na direção sudeste dos ventos ao longo do dia, permitindo fixar as torres nesta orientação geográfica. Grande parte do Submédio do São Francisco se encontra apta para a instalação de centros de geração de energia eólica.

Palavras-Chave: Estações meteorológicas, turbinas eólicas, semiárido.

\section{Introdução}

O maior fornecimento de energia elétrica que é utilizada pela sociedade no Brasil advém de usinas hidrelétricas, que representam $62 \%$ da matriz energética nacional. Existem, ainda, propostas para a construção de novas hidroelétricas nas bacias da Amazônia e do Cerrado. Porém, esse tipo de projeto de implantação de matriz elétrica implica em impactos ambientais relevantes, como perda da biodiversidade nativa (ANEEL, 2015; Martins \& Pereira, 2011).

Com o objetivo de mitigar as ações antrópicas, está em evidência a busca por alternativas energéticas que possibilitem menores impactos sobre a biodiversidade local (Ramos \& Seidler, 2011).

A história das fontes energéticas vem se modificando, principalmente com a escassez dos recursos energéticos não-renováveis e a preocupação com um desenvolvimento sustentável, potencializando o crescimento de novas matrizes, como é o caso da eólica. Nesse contexto, Ramos \& Seidler (2011) perceberam alguns investimentos que vêm sendo realizados e outros que estão previstos no programa de desenvolvimento energético nacional; com todo este investimento, ainda é observada dificuldade na condução dessa tecnologia.

Granero (2012) afirma que o grande potencial para a geração de energia eólica no mundo e a possibilidade da geração de energia em grande quantidade, faz dessa fonte uma alternativa para aumentar a diversidade na matriz energética mundial.

No Nordeste do país, a produção de energia eólica vai crescer, significativamente, nas próximas décadas, principalmente nos estados da Bahia, Ceará e Rio Grande do Norte (Jong et al., 2016). Segundo dados do Operador Nacional do Sistema Elétrico (ONS) (2015), no ano de 2011, apenas $1,8 \%$ da matriz energética eram produzidos utilizando a velocidade do vento e, em 2014, esse valor aumentou para $6,4 \%$.

No Nordeste existe uma grande ocorrência de velocidade média de ventos e de rajadas de ventos. Scruton \& Mewberry (1963) identificaram a importância do efeito das rajadas de ventos através de cálculos de potenciais eólicos, além de considerar as propriedades dinâmicas e a alta frequência, fatores fundamentais para o sucesso na aplicação de projetos eólicos.
Existem estudos variados focando a observação de séries temporais e espaciais de dados baseados em estações meteorológicas. Alguns consistindo de análises espaciais e temporais da velocidade do vento, realizadas utilizando várias abordagens metodológicas (Kavasseri \& Seetharaman, 2009; Shi et al., 2012; Jeong et al., 2014), porém, nenhum realizado em regiões semiáridas.

Além da inexistência de estudos em escalas de mesoregião para o potencial eólico do Nordeste do Brasil, Silva et al. (2002) constataram que não havia nenhum estudo acerca da identificação de tal potencial para uma direção particular do vento, sendo fator primordial para a instalação de turbinas eólicas de grande porte.

A partir dos estudos mais recentes, tem-se que os métodos geoestatísticos, incluindo a krigagem comum, apresentaram ótimos desempenhos quando comparados com outros métodos, sendo utilizados até para cálculos das velocidades de rajada com suficiente precisão (Joyner et al., 2015).

Dentre os fatores limitantes para $o$ investimento na produção de energia eólica no território nacional, se encontra a falta de estudos sobre a viabilidade técnica dos projetos, permitindo a exploração desses recursos renováveis. Diante do exposto, esse estudo tem por objetivo caracterizar a velocidade e a direção predominante do vento para a geração de energia eólica no Submédio São Francisco.

\section{Material e Métodos \\ Área de estudo}

O Submédio São Francisco é formado por municípios pertencentes aos estados de Alagoas, Bahia, Ceará, Paraíba, Pernambuco, Piauí e Sergipe (Figura 1). Segundo a classificação climática de Köppen-Geiger (2007), a região apresenta clima BSwh' (predominando o clima semiárido).

A região apresenta evapotranspiração com valores de $3.000 \mathrm{~mm} \cdot \mathrm{ano}^{-1}$, alta insolação, baixa umidade relativa do ar, pluviosidade média compreendida entre 400 e $600 \mathrm{~mm}^{\mathrm{ano}}{ }^{-1}$, relevo variando de plano a ondulado, com vales muito abertos, altitude variando de 200 a $800 \mathrm{~m} \mathrm{e}$ a vegetação predominante da região é a Caatinga (Cunha et al., 2008). 


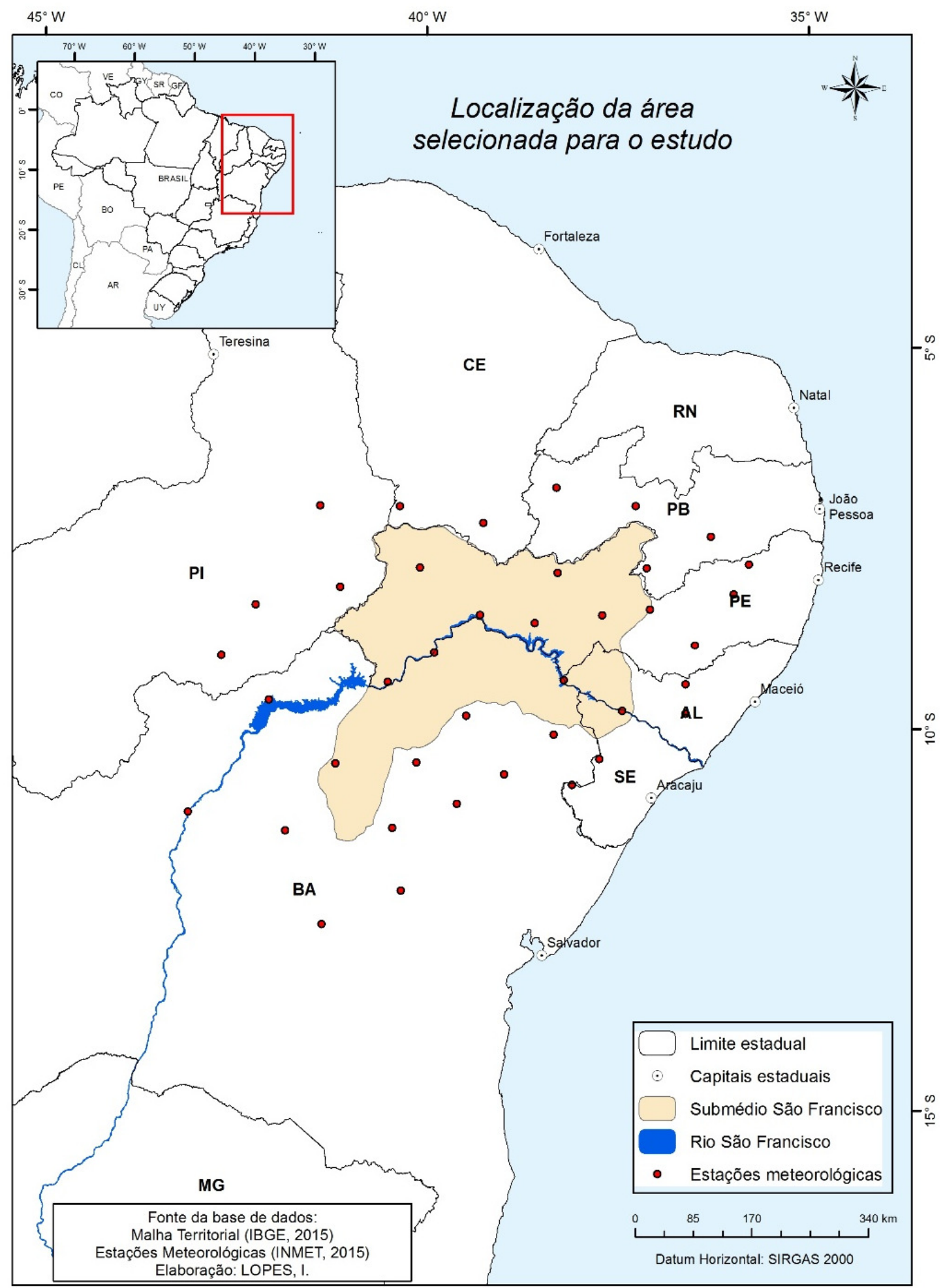

Figura 1. Localização da área de estudo e das estações meteorológicas automáticas do INMET em operação no Submédio São Francisco. Fonte: Autor (2017)..

\section{Fonte dos dados}

Foram utilizados dados horários de velocidade $\left(\mathrm{m} . \mathrm{s}^{-1}\right)$, direção $\left({ }^{\circ}\right)$ e rajada $\left(\mathrm{m} \cdot \mathrm{s}^{-1}\right)$ do vento a $10 \mathrm{~m}$ de altura, temperatura do ar $\left({ }^{\circ} \mathrm{C}\right) \mathrm{e}$ pressão atmosférica $(\mathrm{hPa})$ medidos em 37 estações meteorológicas automáticas do Instituto Nacional de Meteorologia (INMET), modelo Vaisala MAWS301 (Vaisala, 2009), em operação nos municípios Submédio São Francisco e seu entorno (Figura 2).

O período estudado foi de 01-jan-2013 a 31-dez-2016. Os dados de vento foram organizados em dois grupos, o primeiro referente aos dados medidos nos horários entre $6 \mathrm{~h} 00 \mathrm{~min}$ e $17 \mathrm{~h} 59 \mathrm{~min}$ (período diurno) e o segundo aos dados medidos 
nos horários entre $18 \mathrm{~h} 00 \mathrm{~min}$ e $5 \mathrm{~h} 59 \mathrm{~min}$ (período noturno).

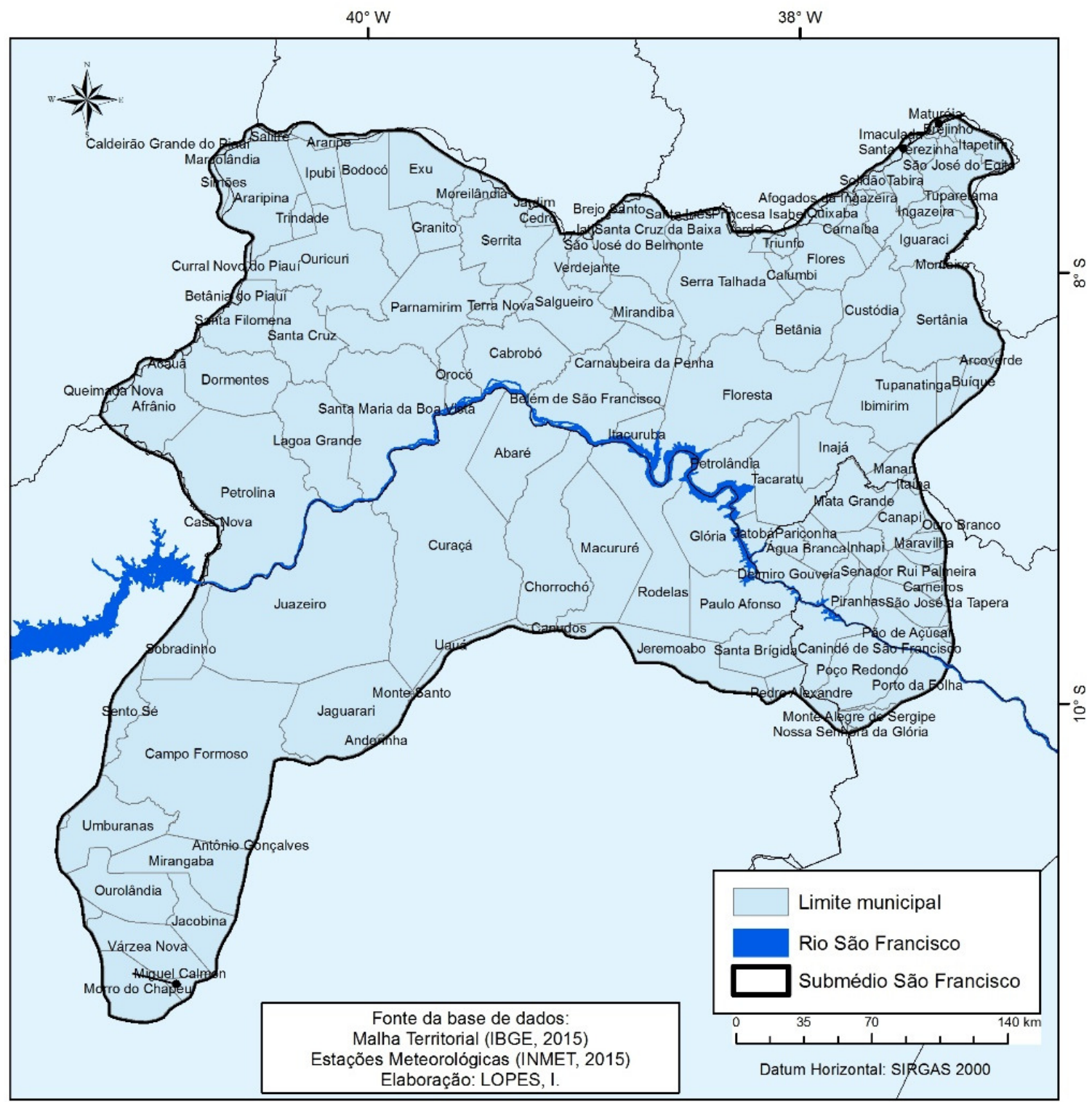

Figura 2. Localização dos municípios abrangidos no Submédio São Francisco. Fonte: Autor (2017).

\section{Cálculos e estimativas}

Com os dados organizados de cada estação em dois períodos, diurno e noturno, foram calculadas as medias aritméticas dos dados de velocidade $\left(\mathrm{m} \cdot \mathrm{s}^{-1}\right)$, direção $\left(^{\circ}\right)$ e rajada $\left(\mathrm{m} \cdot \mathrm{s}^{-1}\right)$ do vento a $10 \mathrm{~m}$ de altura e também da temperatura do ar e da pressão atmosférica.

Os dados de velocidade de vento foram medidos nas estações do INMET a $10 \mathrm{~m}$ de altura. Dessa forma, foi utilizada uma equação logarítmica descrita por Manwell et al. (2002), Equação 1, para estimar a velocidade do vento na altura de $50 \mathrm{~m}$.

$V(Z)=V(Z r) *\left(\frac{\ln \left(\frac{Z}{Z z}\right)}{\ln \left(\frac{Z r}{Z z}\right)}\right)$ onde: $\mathrm{V}=$ velocidade na altura pretendida $\left(\mathrm{m} \cdot \mathrm{s}^{-1}\right)$; $\mathrm{V}(\mathrm{Zr})=$ velocidade na altura de referência $\left(\mathrm{m} . \mathrm{s}^{-1}\right)$; $\mathrm{Z}=$ altura pretendida $(\mathrm{m}) ; \mathrm{Zr}=$ altura observada (m); $\mathrm{Zz}=$ rugosidade, 0,3 .

A qualificação dos ventos foi avaliada considerando a forma de avaliação proposta por Alves (2006), considerando os ventos com velocidades menores que $3,0 \mathrm{~m} \cdot \mathrm{s}^{-1}$ como fracos, entre 3,0 e $5,0 \mathrm{~m} \cdot \mathrm{s}^{-1}$ como moderados e os que possuíam velocidade maior do que $5,0 \mathrm{~m} \cdot \mathrm{s}^{-1}$, como fortes.

Por fim, a potência eólica $\left(\mathrm{W} . \mathrm{m}^{-1}\right)$ por unidade de área dos períodos diurno e noturno de cada estação foram estimados a partir das médias de temperatura do ar $\left({ }^{\circ} \mathrm{C}\right)$, pressão atmosférica (hPa) e velocidade do vento $\left(\mathrm{m} \cdot \mathrm{s}^{-1}\right)$ calculadas seguindo Wagner (1989) (Equação 2). 


$$
P=A *\left(\frac{p}{2 R T} v^{3}\right)
$$

onde: $\mathrm{P}=$ Potêncial eólico $\left(\mathrm{W} \mathrm{m}^{-1}\right) ; \mathrm{A}=$ Área da pá $\left(\mathrm{m}^{2}\right)$, para elaboração deste trabalho a área da pá é igual a $1 \mathrm{~m}^{2} ; \mathrm{v}=$ Velocidade do vento $\left(\mathrm{m} \mathrm{s}^{-1}\right) ; \mathrm{p}=$ Pressão $(\mathrm{Pa}) ; \mathrm{R}=$ Constante especifica dos gases, 287,058 $\left(\mathrm{J} \mathrm{kg}^{-1} \mathrm{~K}^{-1}\right)$; $\mathrm{T}=$ Temperatura $\left({ }^{\circ} \mathrm{K}\right)$.

$\mathrm{Na}$ identificação da direção $\left(^{\circ}\right)$ predominante do vento para os períodos diurno e noturno foram consideradas oito faixas de angulação, definidas pelos pontos cardeais e colaterais.

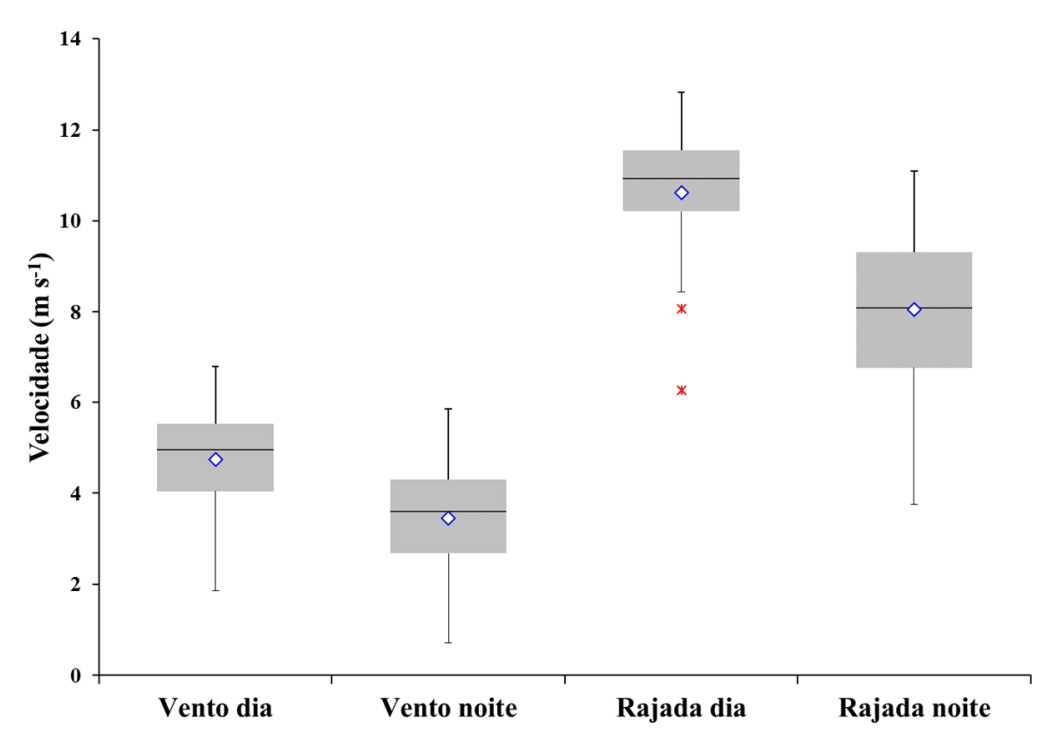

Figura 3. Box-plot das velocidades médias de vento e rajada para dia e noite, para as estações do INMET no período de 2013 a 2016 para a região do Submédio São Francisco.

Observando, também, que ocorre a presença de um ciclo anual em que possui valores máximos no segundo semestre dos anos.

\section{Espacialização dos dados}

Mapas de velocidade, direção, rajada do vento e do potencial eólico do Submédio São Francisco foram confeccionados utilizando a krigagem ordinária de modelo Gaussiano disponível no software Arcgis, versão 10.3.

\section{Resultados}

$\mathrm{Na}$ caraterização geral (Figura 3) observamos os valores da velocidade média dos ventos para o período diurno e noturno de 4,25 e 3,85 m.s ${ }^{-1}$, respectivamente. Do mesmo modo, determinando a velocidade de rajada média dos ventos, detectamos que há maiores valores para o dia e menores para a noite. 
Figura 4. Box-plot das potências de geração de energia eólica para dia e noite, para as estações do INMET no período de 2013 a 2016 para a região do Submédio São Francisco.

Velocidade do vento

Quando observados os valores da velocidade do vento, em termos de média anual, agrupados de forma espacial e temporal, temos diferentes caracterizações. Considerando o período diurno (Figura 5), os municípios incluídos na região com as maiores velocidades de vento, na faixa de 5,15 a 5,65 m.s $\mathrm{s}^{-1}$, foram os de Cabrobó, Dormentes, Lagoa Grande, Curaçá, Afrânio,

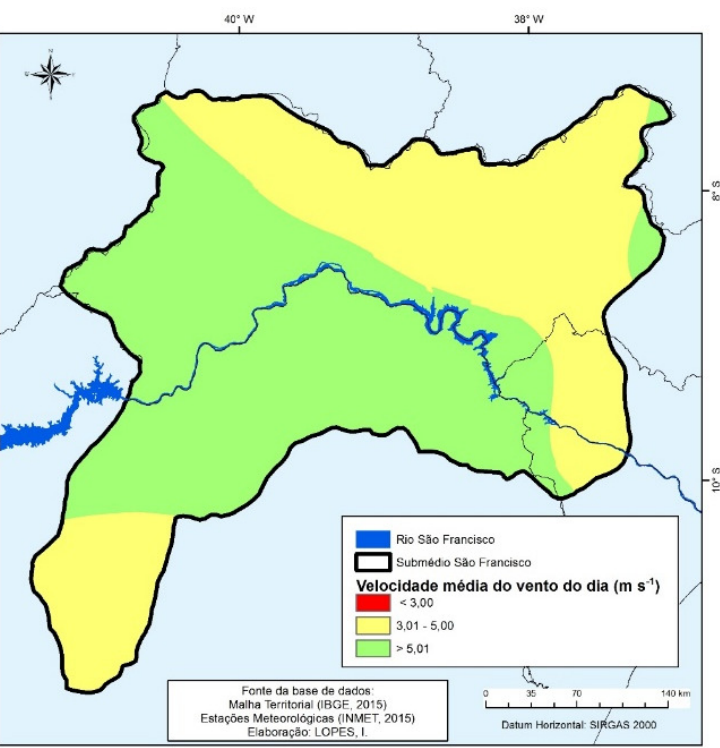

A
Petrolina, Juazeiro e partes dos municípios de Santa Filomena, Santa Cruz, Santa Maria da Boa Vista e Chorrochó. Para o período noturno (Figura 5) observou-se uma mudança com predominância espacial das maiores velocidades dos ventos, na faixa de 5,14 a 5,5 m.s. $\mathrm{s}^{-1}$, destacando-se os municípios de Gloria, Jatobá e Paulo Afonso, partes dos municípios de Macururé, Rodelas, Delmiro Goveia e Jeremoabo.

B

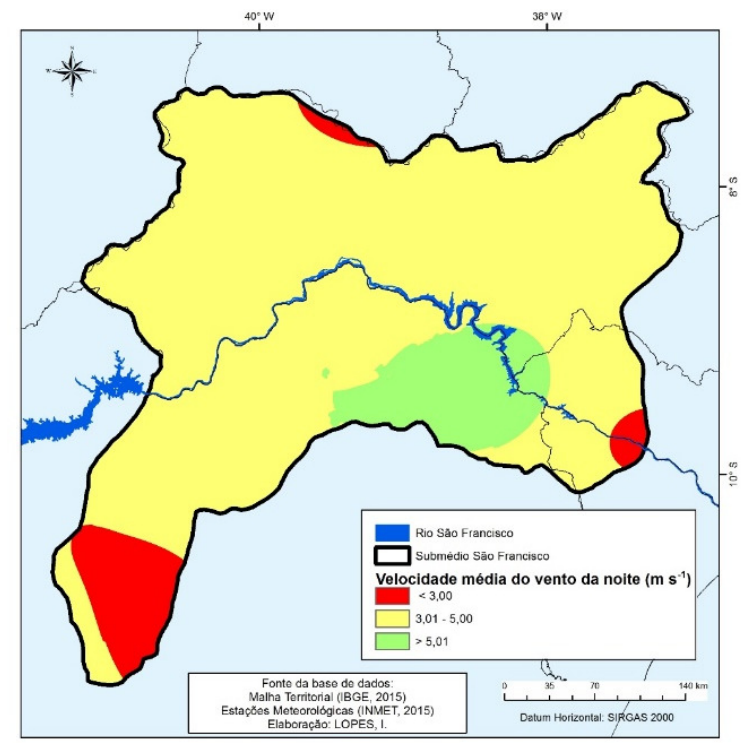

Figura 5. Mapa da distribuição espacial diurno (A) e noturno (B) da velocidade média anual do vento (m. $\mathrm{s}^{-1}$ ), na altura de $10 \mathrm{~m}$, para as estações meteorológicas automáticas do INMET em operação no Submédio São Francisco e municípios ao seu entorno. Fonte: Autor (2017).

O quantitativo de área na faixa de maior velocidade, 5,14 a $5,5 \mathrm{~m} . \mathrm{s}^{-1}$, foi maior para o dia que para a noite no Submédio do São Francisco.

$\mathrm{Na}$ espacialização da velocidade do vento foram obtidos resultados promissores, com a análise em série temporal a $10 \mathrm{~m}$ mostrando claramente a representação dos fenômenos em escalas regionalizadas, indicando melhor a interferência da topografia e da vegetação (Figura $5)$.

\section{Direção do vento}

Apesar de haver uma predominância de sudeste, as direções de ventos são bem variáveis a qual está associada à movimentação da Zona de Convergência Intertropical (ZCIT) e, dessa forma, induz uma previsível sazonalidade nos regimes de vento.
Na Figura 6, os mapas da direção média do vento mostram que a frequência dos ventos que predominam durante os períodos diurnos e noturnos estão no sentido sudeste, indicando que, independentemente de ser dia ou noite, a predominância dos ventos na região do Submédio do vale do São Francisco é de sudeste.

Como pode ser observado no período diurno, no centro da espacialização da direção do vento, nas proximidades da estação meteorológica no município de Cabrobó, ocorre a presença de ventos vindos do sul, que podem ser ocasionados pelo relevo e pelo rio São Francisco.

No período noturno, na parte superior da espacialização, próxima à estação localizada na cidade de Serra Talhada, ocorre a presença de ventos vindos do sul, que podem ser ocasionados pelo relevo da localidade. 
A

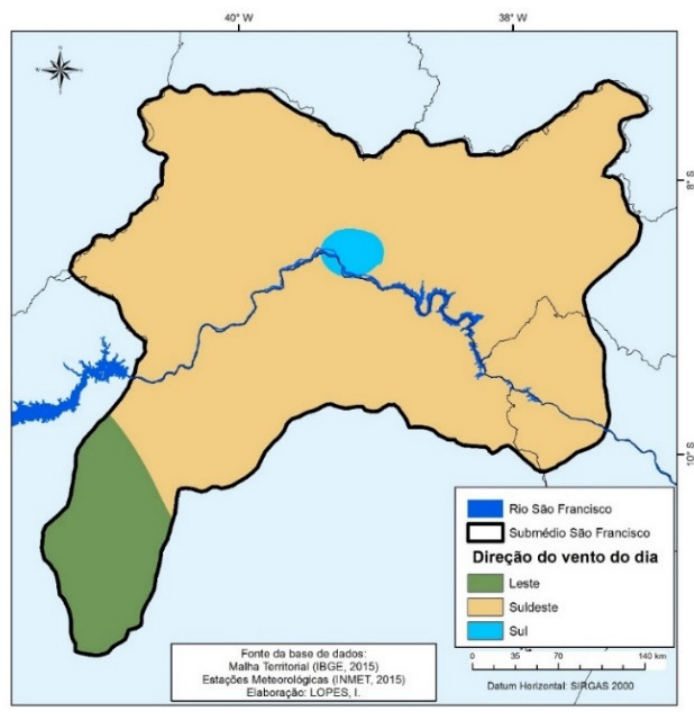

B

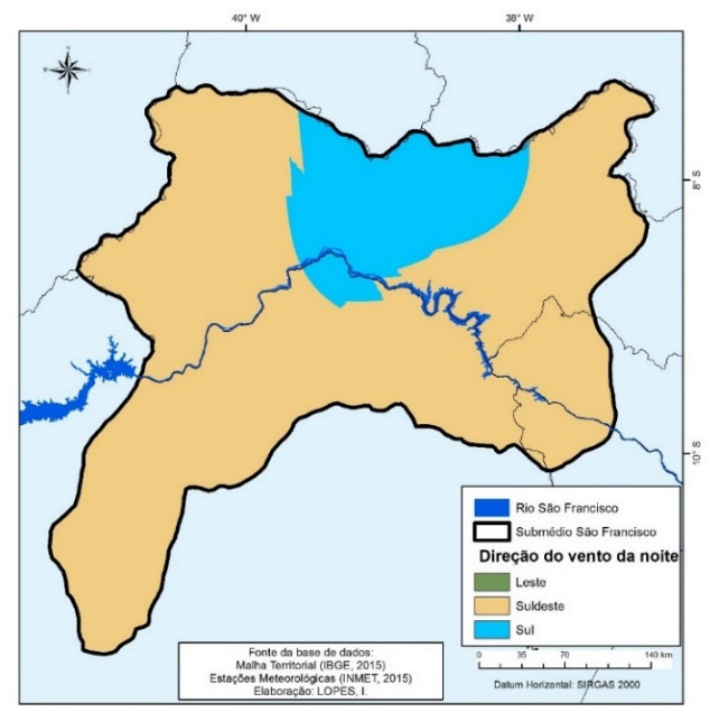

Figura 6. Mapa da distribuição espacial diurno (A) e noturno (B) da direção média anual do vento, para as estações meteorológicas automáticas do INMET em operação no Submédio São Francisco e municípios ao seu entorno. Fonte: Autor (2017).

\section{Rajada dos ventos}

Os picos máximos de velocidade do vento para o período diurno em toda a área atingiram 6,78 $\mathrm{m} . \mathrm{s}^{-1}$, para o período noturno foi de $5,85 \mathrm{~m} . \mathrm{s}^{-1}$.

Os mapas relativos ao ano em que os dados foram analisados mostraram que as rajadas de valores maiores estão predominando sobre os municípios de Dormentes, Petrolina, Afrânio e parte do município de Lagoa Grande, no período diurno (Figura 7A).

Para o período noturno (Figura 7B), existe a predominância da rajada dos ventos nos municípios de Abaré, Chorrochó, Macururé,

A

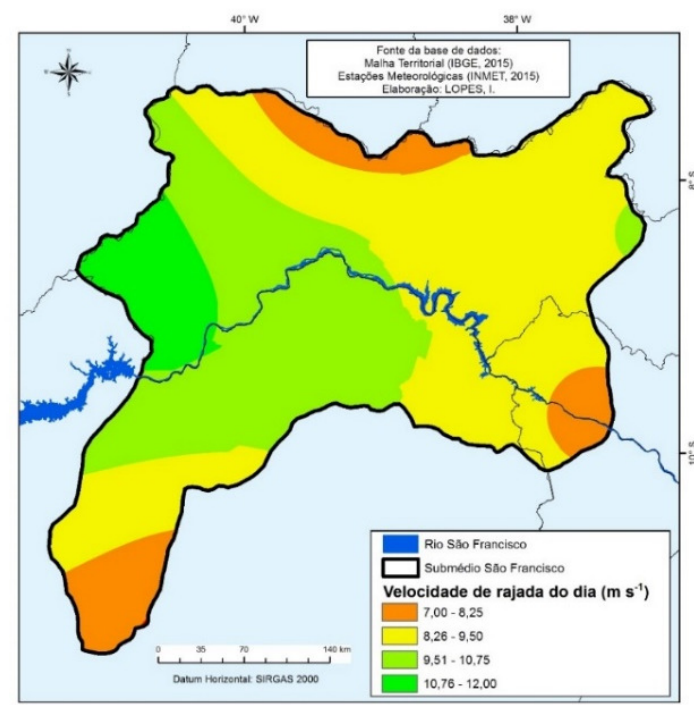

Rodelas, Glória, Paulo Afonso, parte do município de Juazeiro, Curaçá, Petrolina, Lagoa Grande, Santa Maria da Boa Vista, Orocó, Cabrobó, Belém do São Francisco e Jeremuabo.

Alguns desses municípios possuem planos para estudos e possíveis instalações de complexos geradores de energia eólica através da Companhia Hidrelétrica do São Francisco (CHESF, 2016). Dessa forma, este estudo antecipa possíveis áreas para instalação, ou seja, indica potenciais áreas para a instalação de equipamentos e medições mais acuradas.
B

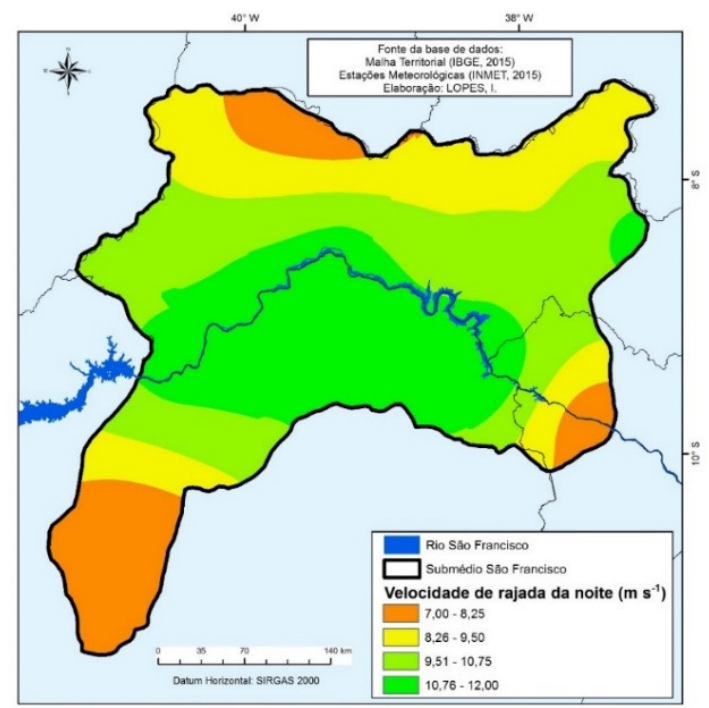

Figura 7. Distribuição espacial diurna (A) e noturna (B) da rajada média anual do vento ( $\left.\mathrm{m} \cdot \mathrm{s}^{-1}\right)$, para as estações meteorológicas automáticas do INMET em operação no Submédio São Francisco e municípios ao seu entorno. Fonte: Autor (2017). 
A região do Submédio São Francisco se caracteriza por apresentar longos períodos de estiagens e por possuir uma matriz energética dependente da água, necessitando da ocorrência de chuvas regulares para a manutenção da produção elétrica. Nesse contexto, a utilização de matrizes de energias alternativas, e que não dependa das chuvas, seria viável para a região, assim como a matriz eólica e/ou a solar.

\section{Potência eólica disponível}

Para o cálculo da potência eólica disponível foram considerados os valores de velocidade do vento $\left(\mathrm{m} . \mathrm{s}^{-1}\right)$, a uma altura de $50 \mathrm{~m}$, obtidos através da transformação nos dados das estações meteorológicas do INMET.

Considerando que os valores de velocidade do vento são diretamente proporcionais ao aumento da altura e que a área das pás utilizadas em aerogeradores são superiores a $1 \mathrm{~m}^{2}$, espera-se que os valores obtidos possam apresentar aumentos consideráveis e, deste modo, viabilizar um projeto de implementação de parques eólicos na região.

A

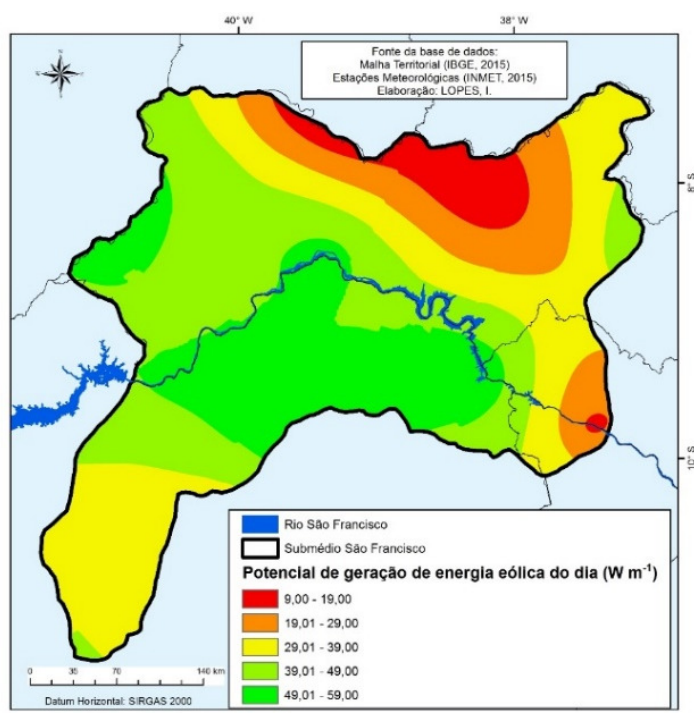

Dentre as áreas estudadas, a classificação que apresentou a menor potência eólica do período estudado foi aquela de $9 \mathrm{~W} \cdot \mathrm{m}^{-1}$ e, por sua vez, a maior potência foi de $59 \mathrm{~W} \cdot \mathrm{m}^{-1}$.

Nas Figuras 8A e B constam os mapas de representação do potencial eólico existente na região. Através da análise dos mapas pode ser observado que os maiores valores de potência eólica estão predominando no período diurno, se destacando os municípios de Dormentes, Lagoa Grande, Curaçá, Petrolina, Afrânio, Pedro Alexandre, parte do município de Santa Filomena, Santa Maria da Boa Vista, Chorrochó, Macururé, Paulo Afonso e Juazeiro.

A metodologia de krigagem aplicada foi eficiente para indicativos de áreas com maior velocidade de vento assim como para a determinação da potência de geração de energia.

Ainda assim, é importante a realização de estudos para uma melhor visualização de erros gerados pela metodologia, sendo necessário para compreender melhor as descontinuidades das classes na espacialização da potência da energia gerada.

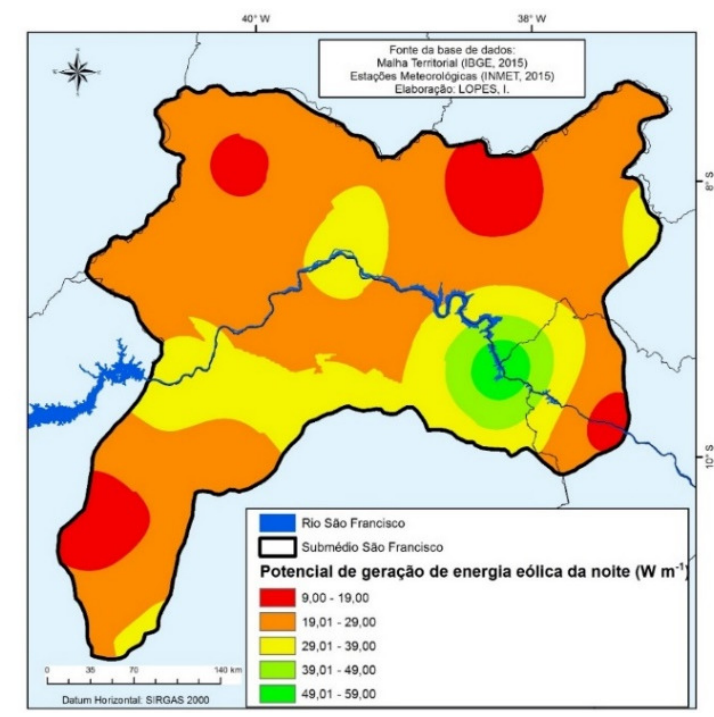

Figura 8. Distribuição espacial diurna (A) e noturna (B) da potência de geração de energia eólica média anual $\left(\mathrm{W} \cdot \mathrm{m}^{-1}\right)$, através as estações meteorológicas automáticas do INMET em operação no Submédio São Francisco e seu entorno. Fonte: Autora (2017).

Nos anos mais recentes, a região Nordeste vem aumentando sua contribuição na produção de energia eólica (CHESF, 2017). Estudos de Parques de Energia Eólica no Submédio São Francisco especificamente, nos municípios de Curaçá, Casa Nova, Sento Sé e Sobradinho.

Segundo Alves (2012), é comum possuir áreas com pouco potencial em uma macro ou mesorregião, assim nas áreas observadas, que possuem um menor potencial de produção de energia, poderiam ser instalados pequenos projetos de aerogeradores para suprir à quantidade de pequenas e médias comunidades que estão longe dos grandes centros urbanos e de certa forma ainda não estão conectados à rede elétrica.

Como mais de $62 \%$ da produção de energia elétrica brasileira é proveniente de usinas hidrelétricas (ANEEL, 2015). Dessa forma o potencial eólico do Submédio São Francisco é favorável em períodos de níveis mínimos de reservatórios, principalmente a barragem de Sobradinho-BA que possui uma grande significância no cenário nacional. Além de que, segundo Alves (2012), participação dessas 
possíveis usinas eólicas no sistema elétrico poderão contribuir para a estabilização sazonal da oferta de energia.

\section{Discussão}

Os valores de velocidade médios do vento para a região do Submédio São Francisco assemelharam-se a estudos na região dos municípios de São Gonçalo, Patos, Monteiro, Campina Grande e João Pessoa na Paraíba-PB, realizados por Lima et al. (2010).

Em comparação com estudo de energia eólica potencial na Corea do Sul, onde há presença de parques eólicos, Um \& Kim (2017) observaram variações longo de gradientes de altitude e agruparam em quatro grupos de potenciais e ainda assim, os maiores valores foram de semelhantes aos observados para a região do Submédio do São Francisco.

Segundo Copel (2007), velocidades do vento a baixo da ordem de 2,5 a $3 \mathrm{~m} \cdot \mathrm{s}^{-1}$ não justificam a implantação de aerogeradores, porque a geração de energia elétrica se inicia com velocidades do vento nessa faixa de valores. O que pode ser aplicado vastamente para o Submédio.

Os picos de velocidades observados são ideais para a utilização de pequenos sistemas isolados, ou seja, para a geração de energia para residência ou para fazendas com sistemas mecânicos para bombeamento de água (Rossi \& Oliveira, 2015).

Estudos no país revelam o potencial para produção de energia com a utilização da velocidade do vento, para corroborar o estudo em questão, onde foram observados valores próximos aqueles encontrados por Souza (1993), ao estudar o potencial eólico do Estado de Minas Gerais $\mathrm{O}$ autor afirmou que a viabilidade de parques eólicos só é obtida com valores de velocidade média dos ventos superiores a $3 \mathrm{~m} \cdot \mathrm{s}^{-1}$. Mais recentemente, Santos (2009), ao avaliar o potencial eólico do campus da Faculdade Assis Gurgaz em Cascavel, no Estado do Paraná, confirmou a viabilidade de geração de energia eólica com valores médios de velocidade do vento inferiores a $3 \mathrm{~m} \cdot \mathrm{s}^{-1}$.

Segundo a proposição de Alves (2006), o submédio possui em sua maioria das áreas estudadas são de ordem moderada para geração de energia eólica, assim justificando a importância da instalação desses aerogeradores.

A mesma tendência de velocidades do vento do Submédio durante o dia serem maiores que a noite foi observado por Munhoz e Garcia (2008) para a região paulista de Ituverava-SP, constatando-se uma superioridade média de médio de $40 \%$.

Oliveira et al. (2012), estudando a influência das variações dos ventos em Mossoró/RN, obtiveram valores médios de velocidade do vento de em torno de $3 \mathrm{~m} \cdot \mathrm{s}^{-1}$. Esses valores são bem inferiores e poucos representativos quando observados os valores de espacialização para o Submédio do São Francisco.

Com a transformação descrita por Manwell et al. (2002) (Equação 1) e com a descrição do processo de transformação, a velocidade seria multiplicada por 1,4589. Dessa forma existe uma elevação representativa na velocidade e consequetemente na dinâmica e potência de geração de energia.

Essa forma de expressão corrobora Souza \& Granja (1997), que afirmam ser esperado que o aumento na altura das torres eólicas, assim como o aumento da área do rotor, proporcione a elevação no potencial de geração de energia.

Os resultados obtidos por Silva et al. (2004) corroboram este estudo; os autores, estudando a direção do vento em direção Nordeste, observaram que a direção predominante do vento, geralmente, varia de Norte a Sul, com predominância das direções Leste e Sudeste

Essa forma é aplicada para o estudo de geração de energia em todo o mundo, a exemplo de Luo et al. (2008) para a Inglaterra e Joyner et al. (2015) para toda Europa.

\section{Conclusão}

Levando em consideração a localização das estações meteorológicas automáticas do INMET para a caracterização do vento para a geração de energia eólica no Submédio São Francisco e os municípios em seu entorno, deve ser utilizado como instrumento para consideração do alto potencial dessa alternativa energética em partes da região.

O município que é mais favorável a instalação de geradores eólicos é Curaçá, no Estado da Bahia, por apresentar um dos maiores valores de velocidade do vento, consequentemente um dos maiores valores de potencia eólica e uma frequência na predominam no sentido sudeste dos ventos durante todo o dia, podem fixar as torres nessa orientação geográfica.

\section{Agradecimentos}

À Universidade Federal do Vale do São Francisco (UNIVASF) e ao Curso de PósGraduação em Engenharia Agrícola (CPGEA), pela disponibilização da estrutura para o desenvolvimento deste estudo, à Coordenação de Aperfeiçoamento de Pessoal de Nível Superior 
(CAPES) e à Fundação de Amparo a Ciência e Tecnologia de Pernambuco (FACEPE), pela concessão da bolsa de estudo. Ao Instituto Nacional de Meteorologia (INMET), pela disponibilização dos dados para a realização do estudo.

\section{Referências}

ALVES, J. J. A. 2006. Estimativa da Potência, Perspectiva e Sustentabilidade da Energia Eólica no Estado do Ceará. Tese de Doutorado, Universidade Federal de Campina Grande, Campina Grande, Paraíba, Brasil. 163p.

ALVES, J. J. A. 2012. Regionalização do potencial eólico no estado do Ceará. Revista Brasileira de Geografia Física, v. 5, n. 2, p. 332-345.

ANEEL, Agência Nacional de Energia Elétrica. 2016. BIG e banco de informações de geração de enrgia elétrica. Disponível em: http://www.aneel.gov.br. Acesso em: 10 fev. 2016.

CAMPOS, M. J. DE; VENDRAMINI, P. S.; SANTOS, R. F.; CHAVES, L. I.; ROSA, H. A.; SOUZA, S. N. M. 2012. Estimativa do potencial eólico no Município de Palotina-Paraná. Revista Acta Iguazu, v. 1, n. 2, p. 94-103.

CHESF. 2017. Relatório da Administração. Disponível em: https://www.chesf.gov.br/relainvest/Documents/R el.Adm.2015.pdf. Acesso em: 11 jun. 2017.

COPEL, Companhia Paranaense de Energia. 2007. Atlas do potencial eólico do estado do Paraná. Curitiba.

CUNHA, T. J. F.; SILVA, F. H. B. B. DA; SILVA, M. S. L. DA; PETRERE, V. G.; SÁ, I. B.; OLIVEIRA NETO, M. B. DE; CAVALCANTE, A. C. 2008. Solos do Submédio do Vale do São Francisco: potencialidades e limitações para uso agrícola. Petrolina: Embrapa Semiárido, 62p.

GRANERO, F. L. Viabilidade de turbinas eólicas de pequeno porte no Brasil. 2012. Graduação em Engenharia Elétrica, Universidade Estadual Paulista, Guaratinguetá, São Paulo, Brasil. 54p.

JEONG, K.; KOO, C.; HONG, T. 2014. An estimation model for determining the annual energy cost budget in educational facilities using SARIMA (seasonal autoregressive integrated moving average) and ANN (Artificial Neural Network). Energy, v. 71, p. 71-79.
JIN, M. U.; KIM, Y. 2017. Estimating potential wind energy from sparsely located stations in a mountainous coastal region. Meteorol. Appl., v. 24, p. 279-289.

JONG, DE P.; KIPERSTOK, A.; SANCHEZ, A. S.; DARGAVILLE, R.; TORRES, E. A. 2016. Integrating large scale wind power into the electricity grid in the Northeast of Brazil. Journal Energy, v. 100, n. 10, p. 401-415.

JOYNER, T. A.; FRIEDLAND C. J.; ROHLI, R. V.; TREVIÑO, A. M.; MASSARRA, C.; PAULUS, G. 2015. Cross-correlation modeling of European windstorms: a kriging approach for optimizing surface wind estimates. Spatial Statistics, v. 13, p. 62-75.

KAVASSERI, R. G.; SEETHARAMAN, K. 2009. Day-ahead wind speed forecasting using Farmuira models. Renewable Energy v. 34, p. 1388-1393.

KÖPPEN, W.; GEIGER, R. 2007. Update world map of the Köppen-Geiger climate classification.

LIMA, F. J. L.; CAVALCANTI, E. P.; SOUZA, E. P. 2010. Avaliação do potencial eólico em cinco regiões do estado da Paraíba. Revista de Geografia, v. 27 , p. $138-153$.

LUO, W.; TAYLOR, M. C.; PARKER, S. R. 2008. A comparison of spatial interpolation methods to estimate continuous wind speed surfaces using irregularly distributed data from England and Wales. Int. J. Climatol. v. 28, p. 947-959.

MANWELL, J. F., MCGOWAN, J. F., ROGERS, A. L. 2002. Wind energy explained. New York: Wiley, 569p.

MARTINS, F. R.; PEREIRA, E. B. 2011. Enhancing information for solar and wind energy technology deployment in Brazil. Journal Energy Policy, v. 39, n. 7, p. 4378-4390.

MUNHOZ, F. C.; GARCIA, A. 2008. Caracterização da velocidade e direção predominante dos ventos para a localidade de Ituverava-SP. Revista Brasileira de Meteorologia, v. 23, n. 1, p. $30-34$.

OLIVEIRA, F. L.; DIAS, V. H. P.; COSTA, E. M.; FILGUEIRA, M. A. ESPÍNOLA SOBRINHO, J. 2012. Influência das variações climáticas na atividade de vôo das abelhas jandairas Melipona subnitida Ducke (Meliponinae). Revista Ciência e Agrotecnologia., v. 43, n. 3, p. 598-603. 
ONS, Operadora Nacional de Sistemas Elétricos. 2016. Histórico da operação e geração de energias. Disponível em: http://www.ons.org.br/home/. Acesso em: 12 fev. 2017.

RAMOS, F. G.; SEIDLER, N. 2011. Estudo da energia eólica para aproveitamento em pequenos empreendimentos. Revista Vivências, v. 7, n. 13, p. 108-127.

ROSSI, P. H. J.; OLIVEIRA, C. P. DE. Centro de Energia Eólica, PUCRS. Disponível em: http://www.pucrs.br/ce-eolica/index.htm. Acesso em: 22 jan. 2015.

SANTOS, R. G.; SANTOS, R. F.; SANTIAGO, W. E.; FIORESE, D. A. 2009. Avaliação do potencial eólico do campus da Faculdade Assis Gurgacz em Cascavel, PR. Revista Cultivando o Saber, v. 2, n. 3, p. 136-150.

SCRUTON, C.; NEWBERRY, C.W. 1963. On the estimation of wind loads for building and structural design. Proceedings of the Institution of Civil Engineers, v. 1, p. 97-126.

SHI, J.; GUO, J.; ZHENG, S. 2012. Evaluation of hybrid forecasting approaches for wind speed and power generation time series. Renewable Sustainable Energy Rev., v. 16, p. 3471-3480.

SILVA, B. B. da; ALVES, J. J. A.; CAVALCANTI, E. P.; DANTAS, R. T. 2002. Potencial eólico da direção predominate do vento no Nordeste do Brasil. Revista Brasileira de Engenharia Agrícola e Ambiental, v. 6, n. 2, p. 295302.

SILVA, B. B. da; ALVES, J. J. A.; CAVALCANTI, E. P.; VENTURA, E. D. 2004. Variabilidade espacial e temporal do potencial eólico da direção predominate do vento no Nordeste do Brasil.. Revista Brasileira de Meteorologia, v. 19, n. 2, p. 189-202.

SOUZA, A; GRANJA, S. C. 1997. Estimativa dos parâmetros "C" e "K" do modelo de Weibull e da direção dos ventos para Campo Grande e Dourados/MS, Brasil. Revista Brasileira de Agrometeorologia, v. 5, n. 1, p. 109-114.

SOUZA, J. W. 1993. Análise climatológica do potencial eólico no Estado de Minas Gerais. Dissertação de Mestrado, Universidade Federal de Viçosa, Viçosa, Minas Gerais, Braszil, 101p.

UM, M. J.; KIM, Y. 2017. Estimating potential wind energy from sparsely located stations in a mountainous coastal region, Meteorological Applications, v. 24, p. 279-289.

VAISALA. Sistema Vaisala HydroMetTM
MAWS3001.
http://www.vaisala.com/en/Pages/default.aspx.
Acesso em: 25 jan. 2015.

WAGNER, C. S. 1989. Velocidade e direção predominante dos ventos no Estado do Paraná. Londrina: Instituto Agronômico do Paraná, 56p. 